\title{
Teacher explanation of physics concepts: a video study
}

\author{
David R Geelan \\ Griffith University \\ d.geelan@griffith.edu.au
}

\begin{abstract}
Video recordings of Year 11 physics lessons were analyzed to identify key features of teacher explanations. Important features of the explanations used included teachers' ability to move between qualitative and quantitative modes of discussion, attention to what students require to succeed in high stakes examinations, thoughtful use of analogies, storytelling and references to the history of science, the use of educational technology and the use of humor. Considerable scope remains for further research into teacher explanations in physics.
\end{abstract}

\section{Introduction}

Teacher explanation is an under-researched field in science education (Geelan, 2012). This seems counterintuitive, since it seems plausible that the ways in which teachers explain concepts in science to students would be one very important key to the effectiveness of science teaching.

The relative paucity of research may be explained to some extent by the 'constructivist turn' in science education research in the 1980s, which for the past few decades has focused enormous amounts of research on student activities and student meaning-making and knowledge construction. While important, this has to some extent taken the focus of research away from the things that effective teachers do in classrooms.

This paper argues that developing strong evidence-based understandings of teacher explanation offers significant potential for enhancing the education of physics teachers. That potential will be realized in teacher education and professional development activities as the features of teacher explanations outlined in the present research project are examined with prospective and practicing physics teachers as 'occasions for reflection' (Geelan, 1997) on the ways in which they develop and deliver explanations to students.

Explanations offered by teachers to students form only one facet of students' learning experiences, of course, with explanations offered to students by other students as well as a wide variety of other learning activities also being important to learning. There is also an increasing number of ways in which various forms of educational technology offer mediated explanations from teachers or other people to students.

This paper, however, is focused on the specific issue of the explanations that teachers offer to students. Such explanations may be given verbally - sometimes in a lecturing mode, but also in whole-class discussions - as well including as diagrams and calculations written on a whiteboard or screen. They may also include demonstrations of apparatus or small experiments conducted by the teacher. The goal of the present study is to establish a typology of teacher explanations in physics, based on video analysis of actual teacher explanations given in classrooms. This typology will be empirically based in the video evidence, and informed by findings and perspectives from the relevant literature.

\section{Literature Review}


Although teacher explanation in physics - and in science education more broadly - remains an under-researched field, there are a number of empirical and theoretical papers that can contribute to an understanding of this facet of teaching.

Wittwer and Renkl (2008) explored reasons why instructional explanations (in general, rather than specifically in science education) might be ineffective, and developed a framework for creating effective explanations. They note that instructional explanations can be divided according to (a) the goals of instruction, (b) whether the explanations are the primary means of instruction or a complement to another strategy and (c) the medium - text or speech (or, although less explicit in their discussion, computer-based) - in which the instruction is given (p. 49).

Based on an extensive review of the available literature (they also acknowledge the relative paucity of relevant research available), Wittwer and Renkl (2008) outlined features for the design of effective instructional explanations, discussed in more detail below.

They also consider some of the reasons why instructional explanations are ineffective, including the need for instructors to have skills and processes for assessing learner's existing understanding as well as what they call 'advanced beliefs about teaching and learning' (p. 59). From the learner's perspective, problems can include lack of engagement and ability to 'calibrate' their own understanding (i.e. to assume they already understand when they do not) and the possession of existing faulty knowledge that impedes the effectiveness of explanations. The theoretical framework of the present paper, outlined below, draws on that of Wittwer and Renkl (2008).

Sevian and Gonsalves (2008) developed a valuable rubric for evaluating explanations in science education. Building on Shulman's (1986) distinctions between pedagogical knowledge, content knowledge and pedagogical content knowledge, they coded the features of verbal explanations given by 32 graduate students, seeking categories such as (1) Engaging the audience, (2) Connecting to prior understanding, (3) Structuring the explanation, (4), Forming images and (5) Presentation style (p. 1448). The rubric they developed, tested and validated scores explanations on a four-point scale for a number of dimensions under each of Shulman's domains of knowledge. While it would have been possible to use Sevian and Gonsalves' (2008) rubric to score the explanations occurring in the present study, the goal was not so much to rank or score some explanations as better or worse than others. Rather, the goal was to explore the features of the explanations offered by teachers in physics classes.

Treagust and Harrison (1999) discussed the issue of explanations in science and science teaching. They noted that secondary school students often confuse explanation with description (Horwood, 1988), and draw on Ruben's (1990, 1993) work on the philosophy of explanations to discuss issues around explanatory frameworks. Treagust and Harrison note that:

- There are important philosophical and epistemological differences between science explanations and science teaching explanations.

- Science explanations are strictly characterized as theory and evidence-driven, use correct scientific terminology and can include analogical models.

- Science teaching explanations differ in rigor, length and detail, involve varying degrees of 'explain how' and 'explain why', are sometimes open-ended, include human agency and can raise new questions as they answer previous questions. (adapted from p. 1158)

A more detailed review of the literature around explanations in science education more generally is available in (Geelan, 2012). For the purposes of this paper, it is the studies specifically relating to explanations in physics education that are most relevant. 
Zacharia (2005) investigated the effect of computer simulations of scientific phenomena on the nature and quality of the explanations offered by science teachers in a postgraduate course on physics content for practicing teachers. Zacharia used the PredictObserve-Explain sequence with the teachers in relation to both the computer-based simulations and more traditional textbook-based assignments on the content, and found that when the teachers interacted with the computer-based simulations the explanations they constructed were richer, more detailed, scientifically more accurate and involved more formal reasoning.

Nashon (2004) recorded the kinds of analogies used by three Kenyan Grade 10 physics teachers. He determined that many of the analogies used were connected to the students' lifeworlds while a number were also anthropomorphic in nature. Nashon prefers teachers to use what he identifies as 'scientific' analogies, in which both the target concept and the analogy fall within the domain of scientific knowledge, however it could be argued that analogies that use features of the students' own life experience to help them to understand the target scientific concepts might be valuable both in enhancing understanding and in keeping students interested in science.

Podolefsky and Finkelstein (2007) offer an approach for building frameworks of linked analogies to scaffold student learning in physics, particularly the learning of difficult, abstract concepts. They present the results of a comparison of the approach they advocate with a non-analogical approach to teaching the same concepts, which shows significant advantages of their approach for students' conceptual learning.

\section{Theoretical Framework}

The theoretical framework for the present study draws on features of the work of Wittwer and Renkl (2008), which in turn draws on both a broad review of psychological studies of instructional explanations and the work of philosophers such as Ruben (1990). The rubric developed by Sevian and Gonsalves (2008), while potentially relevant, was not explicitly drawn on in gathering and analyzing the data in this study.

Wittwer and Renkl (2008) state that instructional explanations should:

- be adapted to the learner's knowledge prerequisites - that is, part of the art and skill of explanation is adapting explanations to the needs of the learner, both in terms of existing knowledge and in terms of cognitive development and capacity. This is a further point of departure from some of the more simplistic notions of 'exemplary' explanations - the notion that excellent explanations can simply be transplanted from one context to another (this is not to deny that there exist more sophisticated notions of exemplary practice that include adaptation to context). An explanation, in order to be an explanation, is given to a specific audience, and adapting the explanation to the audience is a crucial part of explaining.

- focus on concepts and principles - this is related to the work by Treagust and Harrison (2000) in which they analyze explanations given by Nobel Prize-winning physicist Richard Feynman, and identify the way in which Feynman uses principles and axioms of physics in his explanations. Drawing on concepts and principles as the motivation for actions and practices is an important part of physics explanations: teaching physics is to a large extent about teaching students the ways in which the rich explanatory frameworks offered by physics explain their experiences of the physical world. Wittwer and Renkl (2008) review a large number of studies of explanations that fail to focus on concepts and principles and are consequently ineffective. 
- be integrated with the learner's ongoing cognitive activities - drawing on (cognitive) constructivist views of learning that place learners' cognition at the center of discussions of learning, Wittwer and Renkl (2008) focus on learners' active engagement with explanations. Learners should not be passive recipients of explanations but active co-creators, evaluators and processors of explanations in order to maximize their learning effectiveness. In relation to multiple media, interactivity has also been shown to enhance effectiveness, when compared with passive 'consumption' (Mayer, 2005).

- not replace learner's knowledge-construction activities - Wittwer and Renkl (2008) attend to some of the contexts in which offering instructional explanations may do more harm than good. In situations where students already have some knowledge on which to build, it may be much more productive for them to create, test and enhance their own explanations, and explanations offered by teachers have the potential to short-circuit this process. Teacher explanations can lead both teachers and students to over-estimate the extent to which students genuinely understand the content being learned, and a number of studies demonstrated deeper learning based on studentconstructed than teacher-created explanations.

The final element was not really considered in the present study, since the focus was on teacher explanations. Whether or not learning might have occurred more effectively had students instead constructed their own explanations is a fascinating question in need of further research attention in the context of physics education, but falls beyond the purview of this study. The ways in which the first three dimensions of Wittwer and Renkl's framework were observed are discussed below. The present study goes beyond this framework in two ways.

First, the focus is much narrower: Wittwer and Renkl were concerned with all instructional explanations, at all levels of education and in all subject areas. This study is focused on Year 11 physics teachers, so there are specific features of the explanations categorized, such as explicit attention on the part of teachers to the ways in which students will need to be able to demonstrate their understanding of the physics concepts in the looming external high-stakes examination that are not part of Wittwer and Renkl's (2008) much broader framework.

Second, however, the naturalistic coding of features in the video recorded classroom lessons also attended to features that were not really considered in Wittwer and Renkl's (2008) discussion, such as the use of humor and the use of educational technology. In a sense these do fall into areas considered by Wittwer and Renkl, in that educational technology are addition media in which explanations are offered, in primary or secondary roles. Humor may be seen as one form of adaptation of the explanations to the needs and interests of the learners, but was attended to more because it struck the researchers as a common feature of physics teachers' explanations.

Over all, the theoretical framework drawn from Wittwer and Renkl's (2008) paper informed, rather than determined, what was observed, and a naturalistic (Guba \& Lincoln, 1982) approach was taken to the inquiry, linked with a constructivist grounded theory (Charmaz, 2000, 2001) approach to analysis of the data and the development of categories of phenomena.

\section{Method}

This paper reports a study of teaching by 16 Year 11 physics teachers, using video analysis to explore the explanations offered by physics teachers. The 16 teachers in the study taught in Perth, Australia (4 teachers) and Edmonton, Canada (12 teachers). The classrooms in the 
Canadian and Australian contexts were extremely similar. In both jurisdictions students were in Year 11 (aged about 15-17), and being prepared for high-stakes external examinations at the end of their school careers in Year 12. The cultures of the two countries are quite similar, but the culture within physics classrooms was even more similar. Although this is in a sense an international study, no international comparisons are included. There were more differences between classrooms within the Australian and Canadian samples than between the Australian and Canadian cases, so the whole sample has been treated together.

The schools and teachers were chosen on the basis of their past success in helping students achieve high scores in physics. This approach was adopted, despite including some problematic assumptions about the correlation between physics understanding and excess on high-stakes external examinations, for two reasons:

1. Students enter senior physics courses with the expectation of success, an expectation shared by their parents. This is usually defined in terms of good grades and very often in terms of access to particular university courses. It is important that teaching and learning honors these aspirations, while at the same time attempting to foster deep physics learning and understanding on the part of students.

2. Suspicion of 'exemplary teacher' programs and descriptions of 'exemplary teaching', both because teaching is so strongly contextual that contextless prescriptions of excellence seem impossible and because so many of the published studies seem to define the term as 'teaching as the researcher would teach' rather than in terms of any more empirical criterion.

The goal, then, was to use the notion of 'successful teaching', defined by success on a measure that is used by teachers, students and parents, as a 'first cut' to identify schools in which to study pedagogy - specifically teacher explanations. Rather than a single description of 'exemplary practice', the study sought examples of explanatory frameworks and practices that were well adapted to particular contexts and situations, as well as looking for any features that might appear consistently across different contexts.

Different schools within the study had different cultures, drew on different student populations and catered to different aspirations. Some were high-fee private schools that drew from, and prepared students to join, socially advantaged communities. Aspirations in these schools were for very high scores on the external examinations in order to guarantee student access to competitive university programs. Students, teachers and parents were united in these expectations. Other schools were state or Catholic schools in middle class suburbs. Aspirations were similar in a sense - most students reported studying physics for reasons of access to particular university courses rather than for its intrinsic interest and value, for example - but were moderated by other goals and less focused. Yet other schools in the study served urban communities of low socio-economic status, where the focus was on students staying in school and completing Year 12, and the general focus on academic achievement was less distinct amid a number of other issues and imperatives.

Detailed descriptions of the technological solutions used in the project, along with some of the methodological issues around the use of video analysis for this kind of close analysis of classroom practices has been reported elsewhere (Geelan, 2010). Other authors have also explored the affordances of digital video analysis for researching interactions (Pearce, Arnold, Phillips \& Dwan, 2010). In brief, digital video was recorded from the back of the classrooms using a miniDV camcorder and digitized using iMovie and Quicktime on a Mac computer. The video was transcribed and then analyzed using the open source Transana (http://www.transana.org/) video analysis software package, which is available for both Mac and Windows operating systems.

The affordances of classroom video for studying this issue include the ability to watch the same sequence multiple times and to 'over-code' the video on multiple dimensions, but 
the video recording and analysis was considered as a tool for conducting the research rather than as a focus of the research. No comparison was made, for example, between the affordances of video compared with audio-only recording or with other modes of classroom observation such as protocols or checklists. Some discussion of the technical and methodological issues around video analysis in this study has, however, been published elsewhere (Geelan, 2010).

A grounded theory approach (Charmaz, 2000, 2001) was adopted when coding the video lessons. That is, the video was transcribed and repeatedly watched, and themes that occurred in multiple lessons and multiple classrooms listed. A hierarchy of emergent themes and issues was developed, and then each video segment was coded, as described below. The process of constructing categories and coding video was iterative, and was continued until the coding scheme was sufficiently comprehensive to capture all of the key features of the explanations offered in the lessons.

Strategies for enhancing the trustworthiness (Guba, 1981) of the coding of the video and the features identified including showing some of the participating teachers the coded video and checking that they felt the codes adequately captured their intentions and the features of their explanations from their perspectives ('member checking'). Codes and categories were also discussed with research colleagues ('peer debriefing'), in particular in relation to the adequacy and comprehensiveness of category systems and consistency of coding.

Important features of the Transana video analysis software included the ability to code for both single instants and for extended sections of video from a few seconds to a few minutes, as well as the ability for codes to overlap. For example, a particular section of video might exemplify both open-ended questioning and the use of an analogy, and both things needed to be coded for analysis. Specific features coded for - based on the literature included in the literature review above - included:

- the use of analogies and metaphors, with a separate code where teachers paid explicit attention to the ways in which the analogical concept was unlike the target concept (i.e. the places where the analogy breaks down),

- instances where teachers worked through calculations on the board,

- use of diagrams and of electronic animations or simulations,

- use of apparatus and demonstrations in explaining,

- use of anthropomorphic and teleological language,

- teachers' use of hand gestures or body movements to illustrate their explanations,

- appeals to earlier learning in the course,

- drawing on knowledge from other courses the students are taking (usually, but not always, mathematics),

- explicit allusions to assessment such as 'you'll need to know this for the test' or 'this is how I would do this problem in an exam',

- use of questions including open- and closed-ended questioning,

- jokes and humor, including pop-culture allusions.

Time coding could potentially have been used as a means of adding a quantitative dimension to the video analysis - measurement of the proportion of time spent doing calculations on the board, versus helping students with calculations in their seats, for example. This was not done, simply because the video recording of the lessons occurred on a somewhat opportunistic schedule, when it was possible for the researchers to get to the school, and since the focus of the study was on teachers' verbal explanations the camera was often turned off during student experiments or 'seatwork'. This meant that there was no sensible 'timeline' for 
the study in each classroom as a whole to which particular activities could be compared to analyse the proportion of time spent on them. This meant that the study was entirely a qualitative analysis of the features of teacher explanations as they were observed in the recorded classes.

Between 2 and 4 hours of video were recorded in each of the 16 participating classrooms in the study.

Permission was gained from the participating teachers to use the video in teacher education classes, and the analysis has made it possible to create collections of linked vignettes illustrating several different teachers' approaches to, for example, a particular topic in physics. Alternatively, video vignettes can be clustered in relation to a particular common feature of explanations, such as the use of humor or particular approaches to questioning. The use of classroom video has considerable potential to bring the activities of working physics classrooms into university teacher education classes and professional development activities.

\section{Results and Discussion}

The classroom video was recorded at a number of different times across the school year, in both countries. The physics syllabuses in the two jurisdictions were very similar, with most of the topics covered in Year 11 being the same, and because most of the year was covered across the 16 classrooms, most of these topics are represented here. Table 1 lists some features that were seen in common in each school in which a particular topic was taught (most topics in the list were observed in at least two schools and some in as many as four). These features are the kinds of things that can very easily be incorporated in physics teacher education courses, phrased as "many physics teachers use these kinds of strategies to explain these particular phenomena". Student teachers can be encouraged both to learn the relevant knowledge and skills required to use these approaches, and also think creatively about ways to expand their personal physics teaching repertoires.

\begin{tabular}{|l|l|}
\hline Topic & Features of explanations \\
\hline $\begin{array}{l}\text { Kinematics - simple } \\
\text { motion }\end{array}$ & $\begin{array}{l}\text { Use of demonstrations with objects such as balls and } \\
\text { dynamics trolleys moving in the classroom } \\
\text { Use of student experience of sport or other activities, } \\
\text { and students' existing conceptions about motion } \\
\text { Challenge to impulse views of motion }\end{array}$ \\
\hline $\begin{array}{l}\text { Dynamics - motion, } \\
\text { force and energy }\end{array}$ & $\begin{array}{l}\text { Appeal to the idea of 'doing work' and how much work } \\
\text { it takes to achieve a particular result }\end{array}$ \\
\hline $\begin{array}{l}\text { Electricity - static, } \\
\text { current, simple circuits }\end{array}$ & $\begin{array}{l}\text { Use of the idea of 'power' as related to cars and } \\
\text { motorcycles }\end{array}$ \\
\hline $\begin{array}{l}\text { Very persistent (used in every case) analogies between } \\
\text { electric current and water currents } \\
\text { Extensive use of circuit diagrams and explicit attention } \\
\text { to the conventions of drawing and analyzing circuit } \\
\text { diagrams }\end{array}$ \\
\hline
\end{tabular}




\begin{tabular}{|c|c|}
\hline $\begin{array}{l}\text { Waves - types, } \\
\text { calculations }\end{array}$ & $\begin{array}{l}\text { Demonstrations using springs, strings, pipes and } \\
\text { musical instruments } \\
\text { - Use of analogies between easily observed transverse } \\
\text { waves in springs and more difficult-to-visualize } \\
\text { longitudinal (compression) waves }\end{array}$ \\
\hline Gravitation & $\begin{array}{l}\text { - Appeal to students' imagination of being out in space } \\
\text { - Reference to science fiction movies } \\
\text { - Links to circular motion, whichever topic was taught } \\
\text { first } \\
\text { - Reference to the history of science and the } \\
\text { geocentric/heliocentric controversy }\end{array}$ \\
\hline $\begin{array}{l}\text { Circular and Simple } \\
\text { Harmonic Motion }\end{array}$ & $\begin{array}{l}\text { - Demonstrations swinging objects on strings } \\
\text { - Analogies between circular motion and SHM } \\
\text { - Links to planetary orbits and gravitation } \\
\text { - Embarrassed references to an unfamiliar technology } \\
\quad \text { called a 'record player' }\end{array}$ \\
\hline $\begin{array}{l}\text { Light - nature, } \\
\text { reflection, refraction }\end{array}$ & $\begin{array}{l}\text { - Many demonstrations using laser pointers } \\
\text { - Analogies between: - particle nature of light and objects } \\
\text { such as balls - wave nature of light and water waves }\end{array}$ \\
\hline
\end{tabular}

Table One - Distinctive explanatory features related to specific physics topics

Some topics lend themselves to particular explanatory styles more than others. The phenomena of simple motion are much simpler for teachers to demonstrate directly to students in the classroom by dropping, bouncing or throwing objects, for example, whereas the phenomena of electricity tend to be observed 'second-hand' as students read a meter or see a light glow. These differences mean that teachers are much more likely to use analogies to explain the concepts of electricity than of motion, and in fact to use analogies drawn from students' knowledge of motion to help clarify other phenomena.

It is interesting to note from Table 1 that there are few mentions of specific physics concepts and principles identified as features of teacher explanations of specific topics. This is despite the attention of the research team being directed to this issue by the work of both Treagust and Harrison (2000) and Wittwer and Renkl (2008). The latter researchers reviewed a range of literature that demonstrated that explanations that focused on concepts and principles were more effective than those that did not. While these teachers were 'successful' in terms of their students' exam results, then, it could be argued that there was scope for further improvement in the explanations they offered their students. This is a conjecture based on Wittwer and Renkl's work at the moment, and would be amenable to further research investigation.

In addition to the differences based on topics there were differences in the ways in which physics teachers tied physics concepts to the students' everyday lives and experiences. More of the analogies used in the Edmonton classrooms involved ice hockey, or objects sliding on ice. Edmonton is approximately $1000 \mathrm{~km}$ from the coast, so ocean waves and the surf were not often appealed to in the attempt to explain waves, whereas Perth is a beach city and all the students had experience of going to the beach and watching waves.

In addition to some of these differentiated features of the explanations used in the different classrooms, there were a number of features that were general to most or all of the teacher explanations observed. These included: 
1. The 'move to mathematics' - teachers often began explanations of phenomena in a qualitative mode, perhaps with a demonstration or with asking the students to imagine a hypothetical physical situation, but most moved quite quickly to formalize the qualitative information with symbols, units, equations and calculations. A calculation was often delivered as part of an on-going verbal explanation. Some teachers noted that an individual calculation was only one possible example of the phenomenon being explained, while for other teachers this was not always made explicit. This sometimes meant that students struggled to transfer their understanding of the concept to new contexts.

2. Attention to the requirements of success in exams. All the teachers in the study valued physics for its own sake and as a way of seeing the world, but they all also recognized that these students aspired to succeed in physics and gain entry to particular university courses. In both Western Australia and Alberta a large part of this success is predicated on a high-stakes externally administered examination. The schools chosen to participate in the study were those that had been successful on these examinations in the past. That meant that all of the teachers paid explicit attention to this issue, with comments such as "in an exam, I wouldn't set it out this way, I'd do it more simply and quickly". Different teachers did do this in different ways and to different extents, however.

3. Use of analogies. There has been some research attention paid to the use of analogies in physics teaching explanations (e.g. Nashon, 2004; Podolefsky \& Finkelstein, 2007; Thagard, 1992; Treagust \& Harrison, 1999, 2000). All the teachers in the study used analogies extensively. Most were careful to pay attention to the ways in which the analogy worked - the similarities between the analogue and the target concept - but fewer paid careful and explicit attention to where the analogy broke down, or to the differences between the analogue and the target concept. It is possible that this sometimes introduced student misconceptions. Generally, however, analogies were an important facet of teacher explanations, skillfully used.

4. Storytelling and references to the history of science. Many teachers used narratives of particular incidents - such as Archimedes arising from his bath after solving the density problem or the (probably mythical) falling apple observed by Newton - as part of explanations of the related concepts. Fewer used narratives of historical controversies in the history of science such as that between Galileo and the church over whether the earth was at the centre of the solar system or that between Newton and Huygens over whether light was a wave or a particle, however where these debates were described they were accompanied by quite sophisticated ideas about the nature of science and the importance of evidence in deciding scientific questions.

5. Role of technology. Various technologies, from chalk on a chalkboard to ticker-timers, have always been part of explanations in physics teaching, but the use of several newer technologies were notable in the classrooms studied. The advent of very cheap laser pointers has revolutionized the study of light, since it is possible to clearly see the path of a laser beam even in a classroom that cannot be completely blacked out. Laser pointers were used extensively in each classroom in which light was the topic being studied. The advent of data projectors has effected physics teaching explanations in a number of ways. Where the projector is used to delivery a very linear, text-heavy explanation of a phenomenon that students are then asked to copy, it could be argued that this technology is impoverishing physics teaching explanations. Fortunately this phenomenon was rarely observed. More often the data projector was used to bring in video clips to illustrate the phenomena being explained and to show scientific visualisations (animations, and simulations) of both visible and invisible 
(because too large, small, slow, fast or abstract to see) phenomena. In several instances it was also combined with motion sensors or other kinds of 'data probes' to provide immediate graphing of motions in the classroom, with attention being paid to the relationship between a motion and its graph(s).

6. Humor. Different teachers had different personal styles, and physics teacher humor tends to be quite dry, but many of the teachers used humor with their students to help build relationships and improve classroom climate. Some used quite sophisticated 'physics humor' to test and reinforce physics concepts.

There were more specific features of explanations, relating to the ways in which teachers pursued particular questions with students and with where they directed questions to students, and to approaches to classroom organization. Some teachers tended to ask the few students at the front of the room who they knew would provide it for the correct factual answer to their questions and then move on. Teachers in this group tended to ask 'Does anyone have any questions?' after offering an explanation, but not allow any 'wait time' for students to actually respond.

Others conducted extended, almost Socratic, dialogues with the whole class, drawing out their understandings and challenging misconceptions. Teachers in this latter group would often question the same student multiple times, and would intentionally distribute questions to a wide range of students within the class rather than focusing on a few.

Each teacher also had his own professional and personal style and set of knowledge and skills. Some were 'geeks' who reveled in the technologies of science education and the technological implications of physics learning, or who tied the physics concepts to their extensive knowledge of science fiction movies or other pop culture referents. Others possessed large repertoires of anecdotes from the history of science - stories of the lives of scientists or of bizarre experiments and ideas. Still others were more involved in sport, and were able to link the science being learned to their students' sporting interests. The different teachers used humor to different extents and in different ways, based on their own personalities and their relationships with their students.

There are common features in the explanations offered by successful physics teachers, but there is also a huge range of contextual features related to the students, the teacher, the school, the community, the syllabus and a wide variety of other influences that moderates these explanations when they are given in particular instances.

These individual differences underlined the notion that 'best practice' or 'exemplary practice' is a slippery concept. They also fit Wittwer and Renkl's (2008) emphasis on adaptation of the explanation to the learner, although it is not always clear or obvious whether the explanations are being adapted to the specific learners or to the skills, style and preferences of the teachers. A complementary study that focused on a one or two teachers interacting with a variety of classes and students, ideally across a variety of contexts, would help to further elucidate issues of the ways in which explanations are adapted to learners.

The extent to which the explanations given fit Wittwer and Renkl's criterion in relation to 'staying out of the way' of learners' cognition was also difficult to assess in the present study, since the data gathered focused on teacher activities rather than learner cognitive activities. It is possible to draw some inferences from the classroom video, but without closer and more explicit attention to meaning-making on the part of students this must remain an issue for further study.

\section{Conclusion}

A variety of common features were identified in the explanations that physics teachers in Canada and Australia offered to their students in the course of their classroom teaching 
activities. Some of these pertained to verbal explanations and others to teachers' use of whiteboards, demonstrations and information technology to bolster their explanations.

Video analysis is a tool that allows attention to be more easily paid to these other features of teacher explanations than would be possible with audio recordings or classroom observations. Some features of explanations were common to particular topic areas within physics education. There were also a number of idiosyncratic features of explanations identified, linked to the school context as well as to features of teachers and students involved.

Limitations of the study include the fact that there was not a timeline available that would allow some quantitative analyses to be made of the time devoted to explanations versus other teaching activities, and the fact that teachers were observed teaching only one topic.

Teachers were also observed across the school year, so some had been working with the class for up to 9 months and had the opportunity to develop relationships and ways of working with the students, whereas others were recorded at the beginning of the year and were relatively new to their classes. More observation time and a larger range of classes and teachers would have been valuable, of course, but the approximately 40 hours of video recorded, transcribed and analyzed formed a reasonable sample to provide evidence for the identification of the features described in this paper.

Teacher explanations in physics and other science subjects remain badly underresearched, and there is considerable potential for further studies to be conducted. Developing explanations in accordance with Wittwer and Renkl's (2008) framework for constructing instructional explanations, and evaluating them in terms of it, in a systematic way could contribute significantly to the body of knowledge around effective explanations in physics education. Similarly, evaluating explanations using Sevian and Golsalves' (2008) rubric - but even more importantly, introducing beginning and experienced teachers to the features of that rubric, which embody a sophisticated system of research-based knowledge of what constitutes high quality explanations - has the potential to enhance the quality of explanations offered by physics teachers.

Similarly to the ways in which student misconceptions have been studied and categorized in the sciences, there would also be value in collecting, analyzing and categorizing typical teacher explanatory strategies for a variety of common science topics. Such a collection would be a considerable contribution to the education of science teachers. At the same time, recognition that explanations are given by a particular teacher, to a particular class of students, within a particular set of contextual variables relating to the syllabus, the assessment regime, the aspirations of students and parents, features of the school and a plethora of other factors is an important antidote to simplistic prescriptions of 'exemplary practices' in relation to explanation.

The present paper has drawn together a few of the empirical and theoretical threads relevant to discussion of explanation in physics education, and outlined some of the features observed in physics teaching explanations in 16 classrooms. Much more remains to be done. 


\section{References}

Charmaz, K. (2000). Grounded theory: Objectivist and constructivist methods. In N. Denzin \& Y. Lincoln (Eds.), Handbook of qualitative research (2nd ed., pp. 509-535).

Thousand Oaks, CA: Sage.

Charmaz, K. (2001). Qualitative interviewing and grounded theory analysis. In J. Gubrium \& J. Holstein (Eds.), Handbook of interview research: Context and method (pp. 675-694). Thousand Oaks, CA: Sage.

Geelan, D.R. (1997). Epistemological anarchy and the many forms of constructivism. Science \& Education, 6(1-2): 15- 28.

Geelan, D.R. (2003). Teacher expertise and explanatory frameworks in a successful physics classroom. Australian Science Teachers Journal , 49(3), 22- 32.

Geelan, D.R. (2010). Technological and methodological challenges of using classroom video to analyze physics teachers' explanations. International Journal of Multiple Research Approaches, 4(3): 225- 232.

Geelan, D.R. (2012). Teacher Explanations. In B. Fraser, K. Tobin \& C. McRobbie (Eds.), Second International Handbook of Science Education. Dordrecht: Springer.

Geelan, D.R., Wildy, H., Louden, W., \& Wallace, J. (2003). Teaching for understanding and/or teaching for the exam in high school physics. International Journal of Science Education, 26(4), 447- 462.

Guba, E. G. (1981). ERIC/ECTJ annual review paper: Criteria for assessing the trustworthiness of naturalistic inquiries. Educational Communication and Technology: A Journal of Theory, Research, and Development, 29, 75-91.

Guba, E.G. \& Lincoln, Y.S. (1982). Epistemological and methodological bases of naturalistic inquiry. Educational Technology Research And Development, 30(4), 233-252.

Horwood, R.H. (1988). Explanation and description in science teaching. Science Education, 72: 41-49.

Mayer, R.E. (2005). Introduction to multimedia learning. In R. Mayer (Ed.), Cambridge handbook of multimedia learning (pp. 1-16). New York: Cambridge University Press.

Nashon, S.M. (2004). The nature of analogical explanations: high school physics use in Kenya. Research in Science Education, 34(4), 475-502.

Pearce, C., Arnold, M., Phillips, C. \& Dwan, K. (2010). Methodological considerations of digital video observation: beyond conversation analysis. International Journal of Multiple Research Approaches, 4(2), 90-99.

Podolefsky, N.F. \& Finkelstein, N.D. (2007). Analogical scaffolding and the learning of abstract ideas in physics: Empirical studies. Physics Review Studies - Physics Education Research 3, 020104.

Ruben, D-H. (1990). Explaining Explanation. London and New York: Routledge.

Ruben, D-H. (1993). Explanation. Oxford: Oxford University Press.

Shulman, L. (1986) Those who understand: knowledge growth in teaching. Harvard Educational Review, 57, 1-22.

Sevian, H. \& Gonsalves, L. (2008). Analyzing how scientists explain their research: a rubric for measuring the effectiveness of scientific explanations. International Journal of Science Education, 30(11), 1441-1467.

Thagard, P. (1992). Analogy, explanation and education. Journal of Research in Science Teaching, 29(6), 537-544.

Treagust, D.F. \& Harrison, A.G. (1999). The genesis of effective scientific explanations for the classroom (pp. 28-43). In J.J. Loughran (Ed.) Researching teaching: Methodologies and practices for understanding pedagogy. London: Falmer Press. 
Treagust, D.F. \& Harrison, A.G. (2000). In search of explanatory frameworks: an analysis of Richard Feynman's lecture 'Atoms in motion'. International Journal of Science Education, 22(11), 1157-1170.

Wittwer, J. \& Renkl, A. (2008). Why instructional explanations often do not work: a framework for understanding the effectiveness of instructional explanations. Educational Psychologist, 43(1), 49-64.

Zacharia, Z.C. (2005). The impact of interactive computer simulations on the nature and quality of postgraduate science teachers' explanations in physics. International Journal of Science Education, 27(14), 1741-1767. 